\title{
Prognostic significance of head and neck squamous cell carcinoma repair gene polymorphism
}

E. Stur ${ }^{1}$, L.P. Agostini ${ }^{1}$, F.M. Garcia ${ }^{2}$, G.T. Peterle, L.L. Maia ${ }^{1}$, S.O. Mendes ${ }^{1}$, Q.S. Anders' ${ }^{1}$ R.S. Reis ${ }^{1}$, J.A. Santos ${ }^{2}$, D.P. Ventorim ${ }^{2}$, M.B. Carvalho ${ }^{3}$, E.H. Tajara ${ }^{4}$, M. Santos ${ }^{5}$, F. Paula ${ }^{1}$,

A.M.A. Silva-Conforti ${ }^{1,6}$ and I.D. Louro ${ }^{1}$

${ }^{1}$ Programa de Pós-Graduação em Biotecnologia, Universidade Federal do Espírito Santo, Vitória, ES, Brasil

${ }^{2}$ Graduação em Ciências Biológicas, Universidade Federal do Espírito Santo, Vitória, ES, Brasil

${ }^{3}$ Laboratório de Biologia Molecular, Hospital Heliópolis, São Paulo, SP, Brasil ${ }^{4}$ Departamento de Biologia Molecular, Faculdade de Medicina,

São José do Rio Preto, SP, Brasil

${ }^{5}$ Departamento de Medicina, Universidade Federal do Rio Grande do Norte, Campus Caicó, Caicó, RN, Brasil

${ }^{6}$ Departamento de Biologia, Universidade Federal do Espírito Santo, Alegre, ES, Brasil

Corresponding author: I.D. Louro

E-mail: iurilouro@yahoo.com

Genet. Mol. Res. 14 (4): 12446-12454 (2015)

Received February 4, 2015

Accepted June 8, 2015

Published October 16, 2015

DOI http://dx.doi.org/10.4238/2015.October.16.11

ABSTRACT. The aims of this study were to analyze the polymorphisms XRCC1 Arg194Trp, XRCC1 Arg399Gln, XRCC3 Thr241Met, XPC Lys939Gln, ERCC1 Asn118Asn, and RAD51 -98G $>$ C and to verify their influence on radiotherapy response and prognosis of patients with head and neck squamous cell carcinoma (HNSCC). Peripheral blood DNA was extracted from 311 patients and analyzed by PCR-RFLP. Our 
results showed that in irradiated oral and oropharyngeal patients, the 939Gln allele increased 6-fold local disease relapse risk $(\mathrm{OR}=6.04 ; \mathrm{CI}$ $=1.47-24.88)$ and over 2 -fold the earliness of relapse $(\mathrm{HR}=2.63 ; \mathrm{CI}$ $=1.04-6.70)$. As for the XRCC 3 polymorphism, multivariate analysis showed that the $241 \mathrm{Met}$ allele increases over 33-fold local relapse risk $(\mathrm{OR}=33.64 ; \mathrm{CI}=3.23-350.85)$, over 12-fold earliness of relapse $(\mathrm{HR}=$ 12.55; $\mathrm{CI}=2.47-63.73)$ and over 3 -fold earliness of death $(\mathrm{HR}=3.04$; $\mathrm{CI}=1.08-8.61)$. For polymorphism RAD51 -98 , multivariate analysis showed that allele $\mathrm{C}$ increases over 3 -fold the risk of relapse $(\mathrm{OR}=$ $3.13 ; \mathrm{CI}=1.12-8.78)$ and over 2 -fold the earliness of relapse $(\mathrm{HR}=2.84$; $\mathrm{CI}=1.25-6.47$ ). For polymorphism XRCC1 Arg399Gln, multivariate analysis showed that the $399 \mathrm{Gln}$ allele increased the risk of local disease relapse for irradiated oral and oropharyngeal patients $(\mathrm{OR}=3.35 ; \mathrm{CI}=$ 1.10-10.13) by over 3-fold. Based on these results, we suggest that these polymorphisms may be useful markers of prognosis in HNSCC.

Key words: Head and neck cancer; Prognostic outcome; Repair genes; Radiotherapy

\section{INTRODUCTION}

Head and neck squamous cell carcinoma (HNSCC) is a significant cause of mortality and morbidity worldwide, contributing to approximately 540,000 new cases and 271,000 deaths every year, a mortality rate of 50\% (Perez et al., 2006). Main risk factors for HNSCC include alcohol and tobacco use, infections by viruses like human papilloma virus, and individual genetic susceptibility factors (Piccinin et al., 1998; Colombo and Rahal, 2008; Han et al., 2010; Brakenhoff, 2011; Warnakulasuriya et al., 2012).

HNSCC treatment is generally based on surgery, chemotherapy, and radiotherapy. Over the last two decades, several attempts to optimize radiotherapy have been made and some studies have shown that higher and tumor-directed doses might have better results, with little damage to normal tissues (Delaney et al., 2005).

Increased research on the molecular basis of HNSCC has led to better understanding of the molecular pathways involved in radiotherapy response, which allowed the identification of molecular markers that can aid treatment efficacy (Begg et al., 2011). Repair genes, for example, are promising molecular markers because DNA repair is critical for genome preservation and cancer prevention. Polymorphisms in DNA repair genes have been associated with carcinogenesis of several tumors, including tumors from colorectal, lung, and head and neck cancer (Sturgis et al., 1999).

Because of these reasons, we aimed to study the relationship between radiotherapy response in HNSCC patients and the polymorphisms XRCC1 Arg194Trp (rs 1799782), XRCC1 Arg399Gln (rs25487), XRCC3 Thr241Met (rs861539), XPC Lys939Gln (rs2228001), ERCC1 Asn118Asn (rs11615), and RAD51 -98G >C (rs1801320).

\section{SUBJECTS AND METHODS}

We analyzed 311 patients with oral, oropharyngeal and laryngeal squamous cell car- 
cinoma treated at the Head and Neck Surgery Service at Hospital Heliópolis during 20012011. Among these, 151 patients received surgery and irradiation therapy, whereas 160 only received surgery. Results were organized according to tumor type; 210 were oral and oropharyngeal squamous cell carcinoma (SCC) and 101 were laryngeal SCC.

Of the total number of patients, 40 were females and 271 were males, with a mean age of 57 years. For prognosis analysis, all patients were classified according to tumor stage, pathological classification, lymph node presence, local and general disease relapse, and onset of death. In relation to radiotherapy treatment, 151 patients were treated with radiotherapy, of which 102 were patients with oral or oropharyngeal SCC, whereas 49 were laryngeal tumor patients.

Patients were selected for the study based on the following inclusion criteria: a follow up examination after at least 24 months, being metastasis free, and subjected to just surgical treatment prior to sample collection. This study was approved by the Ethics Committee of the Hospital Heliópolis (São Paulo, Brazil), under protocol number 135.

\section{Genotyping}

Peripheral blood DNA was extracted using the salting out method. The target region was amplified by PCR, digested, separated by $2 \%$ agarose gel electrophoresis and stained by ethidium bromide. Table 1 shows primer sequences, fragment sizes, enzymes used, and cycling conditions.

\begin{tabular}{|c|c|c|c|}
\hline Polymorphism & Primer sequence/Fragment & Enzyme & Annealing T/No. of cycles \\
\hline XRCC1 Arg194Trp & $\begin{array}{l}\text { 5'-GCCCCGTCCCAGGTA-3' } \\
\text { 5'-AGCCCCAAGACCCTTTCACT-3' } \\
490 \mathrm{bp}\end{array}$ & PvuII & $57^{\circ} \mathrm{C} / 30 \mathrm{~s}-35 \mathrm{X}$ \\
\hline XRCC1 Arg399Gln & $\begin{array}{l}\text { 5'-TCTCCCTTGGTCTCCAACCT-3' } \\
\text { 5'-AGTAGTCTGCTGGCTCTGG-3' } \\
402 \text { bp }\end{array}$ & MspI & $57^{\circ} \mathrm{C} / 30 \mathrm{~s}-35 \mathrm{X}$ \\
\hline XRCC3 Thr241Met & $\begin{array}{l}\text { 5'-GGTCGAGTGACAGTCCAAAC-3' } \\
\text { 5'-TGCAACGGCTGAGGGTCTT-3' } \\
455 \mathrm{bp}\end{array}$ & $\operatorname{Hin} 1 \mathrm{II}$ & $57^{\circ} \mathrm{C} / 30 \mathrm{~s}-35 \mathrm{X}$ \\
\hline XPC Lys939Gln & $\begin{array}{l}\text { 5'-ACCAGCTCTCAAGCAGAAGC-3' } \\
\text { 5'-CTGCCTCAGTTTGCCTTCTC-3' } \\
281 \mathrm{bp}\end{array}$ & $P v u \mathrm{II}$ & $57^{\circ} \mathrm{C} / 30 \mathrm{~s}-35 \mathrm{X}$ \\
\hline ERCC1 Asn118Asn & $\begin{array}{l}\text { 5'-GCAGACTCACCTGAGGAAC-3' } \\
\text { 5'-GAGGTGCAAGAAGAGGTGGA-3' } \\
199 \text { bp }\end{array}$ & BseMI & $57^{\circ} \mathrm{C} / 30 \mathrm{~s}-40 \mathrm{X}$ \\
\hline RAD51 -98G $>$ C & $\begin{array}{l}\text { 5'-TGGGAACTGCAACTCATCTGG-3' } \\
\text { 5'-GCGCTCCTCTCTCCAGCAG-3' } \\
157 \mathrm{bp}\end{array}$ & $M v a \mathrm{I}$ & $60^{\circ} \mathrm{C} / 30 \mathrm{~s}-40 \mathrm{X}$ \\
\hline
\end{tabular}

The Lilliefors significance test was used to test the normality of the date $(\mathrm{P}<0.05)$. Chi-square was used for association studies and, when necessary, the Fisher exact test was also used. Survival curves were evaluated according to the Kaplan-Meier model and significance was confirmed by the $\mathrm{P}$ value of Wilcoxon. Multivariate-logistic regression and Cox proportional Hazard were used to adjust odds ratio (OD), hazard ratio, and confidence interval values $(95 \% \mathrm{CI})$ of significant results. Clinicobiological relation and a $\mathrm{P}<0.10$ were considered to select the variable to be used in the multivariate analysis. Statistical calculations were done using the Epi Info ${ }^{\circledR}$ v3.4.3 software. 


\section{RESULTS}

After analyzing the genotypes for each polymorphism and general disease relapse, no significant results were found for oral and oropharyngeal SCC in either irradiated or non-irradiated patients. In contrast, for irradiated laryngeal SCC, the XRCC3 polymorphism showed a significant $(\mathrm{P}=0.003)$ relation with general disease relapse, and the Thr allele was more common in cases without relapse. All other general disease relapse analysis showed no significant results.

As for local disease relapse, the XPC gene polymorphism showed a statistically significant relation with irradiated oral and oropharyngeal SCC $(\mathrm{P}=0.018)$, with the presence of the Lys allele being more frequent in cases without local disease relapse. A relation was also observed for the RAD51 polymorphism $(\mathrm{P}=0.042)$ in non-irradiated patients, with the presence of the $\mathrm{G}$ allele being more common in cases without local disease relapse. For all other cases, in patients that either were or were not subjected to irradiation therapy, significant differences were not observed.

In the multivariate analysis, the XRCC1 Arg399Gln polymorphism was associated with the Gln allele and a 3-fold increased risk of local disease relapse in irradiated oral and oropharyngeal patients. A similar relation was observed for the XPC polymorphism, in which a 6 -fold increased risk was observed for the Gln allele (Table 2). Multivariate analysis also showed that the $\mathrm{C}$ allele of the RAD51 -98G $>$ C polymorphism increased the chance of local relapse in nonirradiated patients (Table 2 ) by 3 -fold. We have found a significant relation between laryngeal SCC and XRCC3 $(\mathrm{P}<0.001)$ in patients subjected to irradiation therapy, in which the presence of the Thr allele was more frequent in cases where local relapse was not observed.

Table 2. Multivariate analysis of local disease relapse in oral and oropharyngeal squamous cell carcinoma.

\begin{tabular}{|c|c|c|c|c|}
\hline \multirow[t]{2}{*}{ Variables } & \multicolumn{2}{|c|}{ Irradiated } & \multicolumn{2}{|c|}{ Non-irradiated } \\
\hline & OR $(95 \% \mathrm{CI})^{*}$ & $\mathrm{P}$ & OR $(95 \% \mathrm{CI})$ & $\mathrm{P}$ \\
\hline \multicolumn{5}{|l|}{ Surgical margins } \\
\hline Negative & 1 & & 1 & \\
\hline Positive & $2.47(0.65-9.38)$ & 0.185 & $2.30(0.62-8.49)$ & 0.212 \\
\hline \multicolumn{5}{|l|}{ XRCC1 Arg399Gln } \\
\hline $\mathrm{Arg} / \mathrm{Arg}$ & 1 & & - & - \\
\hline $\mathrm{Arg} / \mathrm{Gln}+\mathrm{Gln} / \mathrm{Gln}$ & $3.35(1.10-10.13)$ & 0.033 & - & - \\
\hline \multicolumn{5}{|l|}{ XPC Lys939Gln } \\
\hline Lys/Lys_Lys/Gln & 1 & & - & - \\
\hline $\mathrm{G} \ln / \mathrm{Gln}^{-}$ & $6.04(1.47-24.88)$ & 0.013 & - & - \\
\hline \multicolumn{5}{|l|}{ RAD51 - 98G $>C$} \\
\hline GG & - & - & 1 & \\
\hline $\mathrm{GC}+\mathrm{CC}$ & - & - & $3.13(1.12-8.78)$ & 0.030 \\
\hline
\end{tabular}

*OR and CI were adjusted by the multivariaté-logistic regression.

Multivariate analysis of laryngeal cancer showed that in irradiated individuals, the presence of the Met allele of the XRCC3 Thr241Met polymorphism increased the risk of local disease relapse by 33-fold $(\mathrm{OR}=33.64 ; \mathrm{CI}=3.23-350.85 ; \mathrm{P}=0.003)$. Death analysis showed no statistically significant differences.

Local disease-free survival analysis in irradiated oral and oropharyngeal SCC patients showed a significant association with the XPC Lys939Gln polymorphism $(\mathrm{P}=0.038$; Figure 1). The survival curve indicated that in the first 12 months after treatment, $30 \%$ of patients with the Gln allele underwent local disease relapse, whereas only $10 \%$ of Lys allele patients 
relapsed. Multivariate analysis also showed that homozygous Gln alleles increased the early onset of local disease relapse by over 2 -fold $(\mathrm{HR}=2.63 ; \mathrm{CI}=1.04-6.70 ; \mathrm{P}=0.042)$.

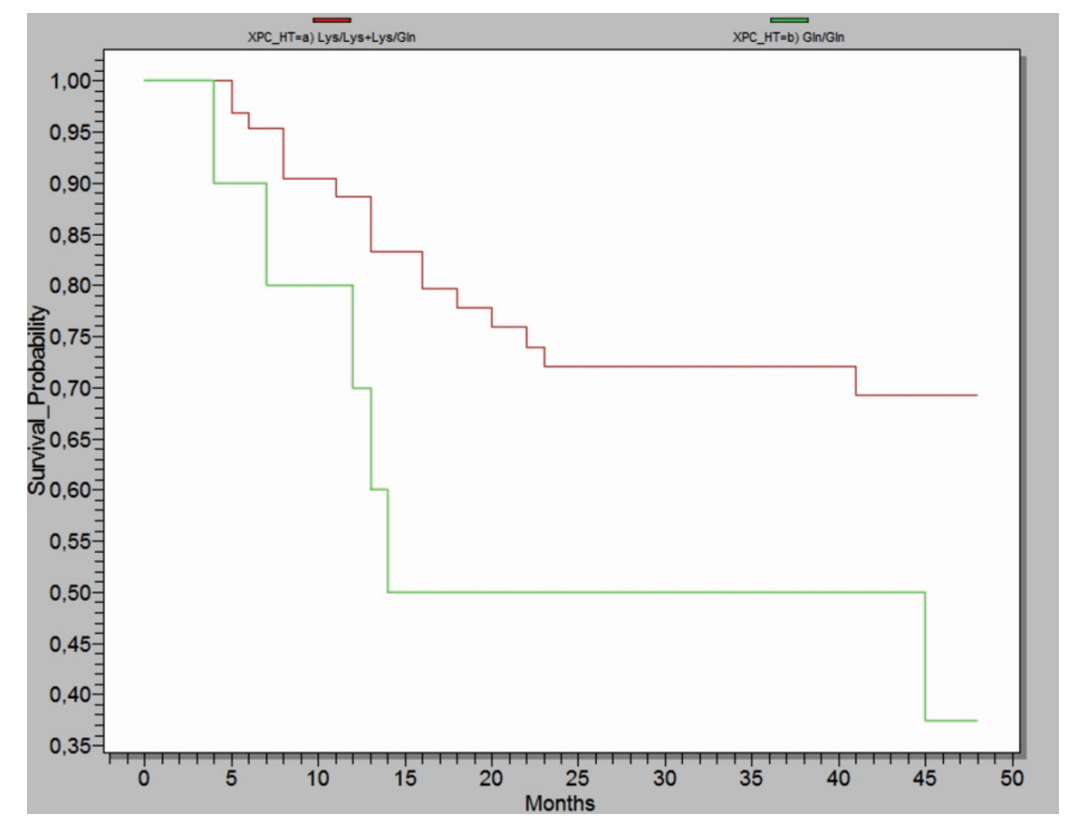

Figure 1. Local disease-free survival curve for the XPC Lys939Gln polymorphism $(\mathrm{P}=0.038)$ in irradiated oral and oropharyngeal squamous cell carcinoma patients $(\mathrm{N}=24)$.

In contrast, local disease-free survival in non-irradiated oral and oropharyngeal SCC patients showed a relation to the RAD51 $-98 \mathrm{G}>\mathrm{C}$ polymorphism $(\mathrm{P}=0.009)$ : approximately $15 \%$ of the occurrences of local relapse in a period of 12 months after surgery was in patients with the $\mathrm{G}$ allele, while $45 \%$ of the $\mathrm{C}$ allele bearers showed disease relapse during the same period. This analysis also indicated that the $\mathrm{C}$ allele increases the early onset of local disease relapse by over 2-fold when compared to the $\mathrm{G}$ allele $(\mathrm{HR}=2.84 ; \mathrm{CI}=1.25-6.47 ; \mathrm{P}=0.013)$.

Local disease-free survival was also evaluated for irradiated laryngeal SCC cases. Polymorphism XRCC3 Thr241Met $(\mathrm{P}<0.001)$ showed a significant relation to local diseasefree survival in irradiated patients, in which, at 12 months after surgery, $30 \%$ of the Met allele bearers had local disease relapse, as compared to $10 \%$ of the Thr allele patients. This analysis also showed that the Met allele presence increased the early onset of local disease relapse by 12-fold when compared to the $\mathrm{Thr}$ allele $(\mathrm{HR}=12.55 ; \mathrm{CI}=2.47-63.73 ; \mathrm{P}=0.002)$.

Disease-specific survival analysis in irradiated laryngeal SCC patients indicated that the XRCC3 Thr241Met polymorphism was significantly related to survival $(\mathrm{P}=0.046)$. Figure 2 shows that 12 months after surgery, $30 \%$ of the Met allele bearers had died, whereas only $10 \%$ of patients with at least one Thr allele showed the same fate. Multivariate analysis demonstrated that Met allele homozygosity was related to worse disease-specific survival, increasing the early onset of disease-specific death by over 3 -fold when compared to that observed with the Thr allele $(\mathrm{HR}=3.04 ; \mathrm{CI}=1.08-8.61 ; \mathrm{P}=0.036)$. 


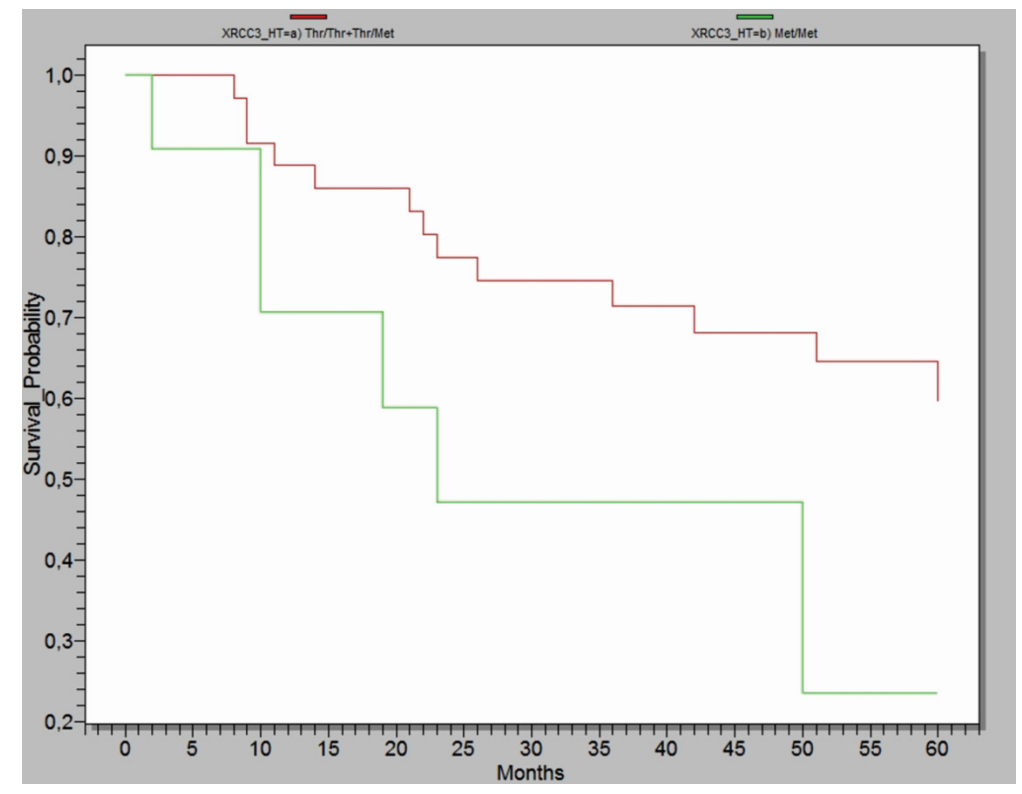

Figure 2. Disease-specific survival curve for the XRCC3 Thr241Met polymorphism in irradiated laryngeal squamous cell carcinoma patients $(\mathrm{P}=0.046 ; \mathrm{N}=19)$.

\section{DISCUSSION}

The present study is an analysis of oral, oropharyngeal and laryngeal SCC patients, with the goal of understanding the importance of DNA repair gene polymorphisms in the outcome of radiotherapy treatment. The XRCC1 Arg399Gln polymorphism allowed us to identify the presence of the $399 \mathrm{Gln}$ allele, which increases local disease relapse risk in irradiated oral and oropharyngeal patients.

The Arg399 allele is related to a worse response to radiotherapy treatment and reduced survival in Asian patients (Borchiellinni et al., 2012). Other studies have shown that the $399 \mathrm{Gln}$ allele is related to worse survival and decreases tumor regression, which is similar to our results. There has also been a report of a better treatment response in heterozygous patients (Borchiellinni et al., 2012).

Wu et al. (2006), working with esophageal SCC treated with chemotherapy prior to surgery, found that $399 \mathrm{Gln}$ allele bearers showed an increased risk of death when compared to patients with the Arg/Arg genotype, who had a longer mean survival. In the present study, the $399 \mathrm{Gln}$ allele increased the occurrence of local disease relapse and therefore, the risk of death. A possible mechanism to explain this result is that the XRCC1 gene repairs DNA by excision repair, and the Gln variant causes a decreased repair ability and, consequently, greater chromosomal damage. Therefore, worse prognosis may be related to genetic instability caused by the radiotherapic treatment, since genetic instability is related to worse prognosis and higher local disease relapse (Wang et al., 2010).

The XRCC1 Arg399Gln polymorphism is located at a critical region for base excision repair, and is needed for recruiting PARP1 by XRCC1. We suggest that the substitution 
of an arginine for a glutamine prevents the formation of secondary structures like the alpha helix, which is important for the protein-protein interactions of the BRCT1 domain, thereby compromising DNA repair (Gao et al., 2010). It is also possible that the 399Gln allele hinders the interaction between XRCC1 and other proteins, resulting in the inefficient removal of radiation-induced DNA damage.

Our results indicate that the $399 \mathrm{Gln}$ allele is associated with poor survival of irradiated patients, which suggests that the Gln allele is associated with poor response to radiotherapy and poor prognosis of local disease relapse. The predicted increase in radiotherapy sensitivity due to reduced DNA repair in patients with the Gln allele was not observed in our study. Our hypothesis is that although this allele is associated with reduced DNA repair, this reduction is not enough to cause cell death and therefore may lead to the accumulation of mutations and increase tumor evolution. Furthermore, the base excision repair pathway works by correcting single-strand breaks that are less lethal than the double-strand breaks normally caused by radiotherapy. This mechanism supports the idea that the XRCC1 399Gln allele contributes to the accumulation of mutations and increases tumor evolution (Komar, 2007).

After analysis of the XPC Lys939Gln polymorphism in irradiated oral and oropharyngeal SCC patients, we found that the presence of the Lys939 allele was related to a lower rate of local disease relapse. Multivariate analysis indicated that the presence of the homozygous 939Gln genotype increased the risk of local disease relapse by 6-fold and the risk of early local relapse by 2-fold when compared to that observed in the presence of the Lys allele.

Yang et al. (2013) analyzed patients with esophageal SCC and found that the Gln/Gln genotype is a risk factor for death and relapse after chemotherapy, and that Gln/Gln genotype bearers respond poorly to chemotherapy when compared to other genotypes, therefore resulting in a worse prognosis. Yang's results are compatible with ours. We showed that the presence of the Lys939 allele had a protective effect and Yang shows that the presence of the Gln allele increased chances of disease relapse.

Little is known about the mechanism by which the XPC Lys939Gln polymorphism alters disease prognosis. The change of a basic and hydrophilic amino acid (lysine) for a neutral amino acid (glutamine) may cause unknown structural and functional protein modifications (Rouissi et al., 2011; Ji et al., 2012). In support of this hypothesis, the 939Gln allele is associated with reduced nucleotide excision repair in vitro (Vodicka et al., 2004; Wang et al., 2010), which is similar to what has been observed for the XRCC1 Arg399Gln polymorphism in which the reduced DNA repair allele is the same that is associated with a worse response to treatment.

Analysis of the XRCC3 Thr241Met polymorphism indicated an association of the Thr241 allele with local and regional relapse in irradiated laryngeal SCC. Multivariate analysis indicated that this allele was related to higher local disease relapse and earlier onset of relapse and death.

There is little information in the literature about the relation between XRCC3 gene polymorphisms and radiotherapy response. Li et al (2006) studied pancreatic cancer, but did not find an association between XRCC3 polymorphisms and global survival. Similarly, Vodicka et al. (2007) did not find any significant differences. In spite of these results from previous studies, the XRCC3 polymorphisms act on homologous recombination and double-strand DNA repair, which are commonly caused by radiotherapy. Our results suggest that these polymorphisms are potential marker of radiosensitivity and that the Met allele is related to worse 
prognosis, since it is associated with the earliness of local relapse disease.

The 241Met allele is associated with defective DNA repair after X-ray-induced damage (Au et al., 2004). Other studies, such as the one by Kietthubthew et al. (2006) and the one by Bastos et al. (2009), suggested that the variant allele increases risk of oral and thyroid cancer.

The XRCC3 gene product stabilizes the RAD51 protein, an essential step in homologous recombination after radiotherapy. The XRCC3 gene segment that encompasses amino acids 63 to 346 is the region that binds to RAD51, corresponding to residues Tyr139 and Phe249 that are needed for this interaction. After the substitution of threonine by methionine at position 241, interaction with RAD51 is compromised, affecting DNA repair (Bastos et al., 2009). The RAD51 G allele was associated with less local disease relapse in non-irradiated oral and oropharyngeal SCC, and multivariate analysis indicated a 3-fold increase in the risk for local relapse and more than a 2-fold increase in the risk for the early onset of relapse when the $\mathrm{C}$ allele was present.

Li et al. (2006) analyzed pancreatic cancer treated with radiotherapy and did not find any association between this polymorphism and survival. Likewise, Gordon et al. (2006) studied rectal cancer and did not report an association of this polymorphism with disease relapse, lymph node status or chemotherapy response, which concurs with our results. We found that the $\mathrm{C}$ allele is associated with worse prognosis, but this is not related to radiotherapy.

Presence of the $\mathrm{C}$ allele is known to increase promoter activity and should also increase DNA repair; however, increased promoter activity resulted in decreased DNA repair due to abnormal RAD51 function, which explains the worse prognosis observed in the presence of the C allele (Borchiellini et al., 2012; Shin et al., 2008; Wang et al., 2013). As for the other polymorphisms (ERCC1 Asn118Asn and XRCC1 Arg194Trp), this study did not find statistically significant associations.

Considering the anatomic sites affected by specific gene polymorphisms, the XRCC1 $399 \mathrm{Gln}$ and XPC 939Gln alleles were related to worse prognosis in irradiated oral and oropharyngeal SCC and the XRCC3 241Met allele was related to worse prognosis in irradiated laryngeal SCC. Our results suggest that decreased DNA repair may not be associated with increased radiosensitivity, and may therefore result in a worse prognosis. Based on these observations, we propose that an efficient repair system may be needed to trigger apoptosis after radiotherapy, in order to prevent the accumulation of mutations and to increase the response to treatment.

\section{CONCLUSION}

Our results show that XRCC3 241Met is associated with a worse prognosis in irradiated laryngeal SCC, and that XPC 939Gln and XRCC1 399Gln are associated with a worse prognosis in irradiated oral and oropharyngeal SCC. Our results also show that the $\mathrm{C}$ allele of RAD51 is related to a worse prognosis in non-irradiated oral and oropharyngeal SCC. Therefore, we suggest that the XRCC1 399Gln, XPC 939Gln, XRCC3 241Met, and RAD51 $-98 \mathrm{G}>\mathrm{C}$ alleles may be used as genetic markers for poor prognosis in oral, pharyngeal and laryngeal tumors.

\section{Conflicts of interest}

The authors declare no conflict of interest. 


\section{ACKNOWLEDGMENTS}

Research supported by Conselho Nacional de Desenvolvimento Científico e Tecnológico (CNPq), Fundação de Amparo à Pesquisa do Estado do Espírito Santo (FAPES) and Coordenação de Aperfeiçoamento de Pessoal de Nível Superior (CAPES).

\section{REFERENCES}

$\mathrm{Au}$ WW, Navasumrit P and Ruchirawat M (2004). Use of biomarkers to characterize functions of polymorphic DNA repair genotypes. Int. J. Hyg. Environ. Health 207: 301-313.

Bastos HN, Antão MR, Silva SN, Azevedo AP, et al. (2009). Association of polymorphisms in genes of the homologous recombination DNA repair pathway and thyroid cancer risk. Thyroid 19: 1067-1075.

Begg AC, Stewart FA and Vens C (2011). Strategies to improve radiotherapy with targeted drugs. Nat. Rev. Cancer 11:239-253.

Borchiellini D, Grimaldi MCE, Thariat J and Milano G (2012). The impact of pharmacogenetics on radiation therapy outcome in cancer patients. A focus on DNA damage response genes. Cancer Treat. Rev. 38: 737-759.

Brakenhoff RH (2011). Cancer. Another NOTCH for cancer. Science 333: 1102-1103.

Colombo J and Rahal P (2009). Genética de cancer de cabeça e pescoço. Rev. Soc. Bras. Cancerol. 55: 165-174.

Delaney G, Jacob S, Featherstone C and Barton M (2005). The role of radiotherapy in cancer treatment: estimating optimal utilization from a review of evidence-based clinical guidelines. Cancer 104: 1129-1137.

Gao R, Price DK, Dahut WL, Reed E, et al. (2010). Genetic polymorphisms in XRCC1 associated with radiation therapy in prostate cancer. Cancer Biol. Ther.10: 13-18.

Gordon MA, Gil J, Lu B, Zhang W, et al. (2006). Genomic profiling associated with recurrence in patients with rectal cancer treated with chemoradiation. Pharmacogenomics 7: 67-88.

Han S, Chen Y, Ge X, Zhang M, et al. (2010). Epidemiology and cost analysis for patients with oral cancer in a university hospital in China. BMC Publ. Health 10: 196-208.

Ji G, Lin Y, Cao SY, Li LZ, et al. (2012). XPC 939A >C and 499C > T polymorphisms and skin cancer risk: a meta-analysis. Asian Pac. J. Cancer Prev. 13: 4983-4988.

Kietthubthew S, Sriplung H, Au WW and Ishida T (2006). Polymorphism in DNA repair genes and oral squamous cell carcinoma in Thailand. Int. J. Hyg. Environ. Health 209: 21-29.

Komar AA (2007). Silent SNPs: impact on gene function and phenotype. Pharmacogenomics 8: 1075-1080.

Li D, Frazier M, Evans DB, Hess KR, et al. (2006). Single nucleotide polymorphisms of RecQ1, RAD54L, and ATM genes are associated with reduced survival of pancreatic cancer. J. Clin. Oncol. 24: 1720-1728.

Perez-Ordonez B, Beauchemin M and Jordan RC (2006). Molecular biology of squamous cell carcinoma of the head and neck. J. Clin. Pathol. 59: 445-453.

Piccinin S, Gasparotto D, Vukosavljevic T, Barzan L, et al. (1998). Microsatellite instability in squamous cell carcinomas of the head and neck related to field cancerization phenomena. Br. J. Cancer 78: 1147-1151.

Rouissi K, Bahria IB, Bougatef K, Marrakchi R, et al. (2011). The effect of tobacco, XPC, ERCC2 and ERCC5 genetic variants in bladder cancer development. BMC Cancer 11: 101-108.

Shin A, Lee KM, Ahn B, Park CG, et al. (2008) Genotype-phenotype relationship between DNA repair gene genetic polymorphisms and DNA repair capacity. Asian Pac. J. Cancer Prev. 9: 501-505.

Sturgis EM, Castillo EJ, Li L, Zheng R, et al. (1999). Polymorphisms of DNA repair gene XRCC1 in squamous cell carcinoma of the head and neck. Carcinogenesis 20: 2125-2129.

Vodicka P, Kumar R, Stetina R, Sanyal S, et al. (2004). Genetic polymorphisms in DNA repair genes and possible links with DNA repair rates, chromosomal aberrations and single-strand breaks in DNA. Carcinogenesis 25: 757-763.

Vodicka P, Stetina R, Polakova V, Tulupova E, et al. (2007). Association of DNA repair polymorphisms with DNA repair functional outcomes in healthy human subjects. Carcinogenesis 28: 657-664.

Wang F, He Y, Guo H, Li J, et al. (2010). Genetic variants of nucleotide excision repair genes are associated with DNA damage in coke oven workers. Cancer Epidemiol. Biomarkers Prev. 19: 211-218.

Wang M, Chu H, Zhang Z and Wei Q (2013). Molecular epidemiology of DNA repair gene polymorphisms and head and neck cancer. J. Biomed. Res. 27: 179-192.

Warnakulasuriya S, Dietrich T, Bornstein MM, Casals Peidró E, et al. (2012). Oral health risks of tobacco use and effects of cessation. Int. Dent. J. 60: 7-30.

Wu X, Gu J, Wu TT, Swisher SG, et al. (2006). Genetic variations in radiation and chemotherapy drug action pathways predict clinical outcomes in esophageal cancer. J. Clin. Oncol. 24: 3789-3798.

Yang PW, Hsieh CY, Kuo FT, Huang PM, et al. (2013). The survival impact of XPA and XPC genetic polymorphisms on patients with esophageal squamous cell carcinoma. Ann. Surg. Oncol. 20: 562-571. 